\title{
Précis of The Primacy of Metaphysics
}

\author{
Christopher Peacocke ${ }^{1}$
}

Accepted: 8 September 2020/Published online: 17 September 2020

(C) Springer Nature B.V. 2020

The goal of The Primacy of Metaphysics (henceforth $P M$ ) is to investigate the relations between the metaphysics of a given domain and concepts of entities in that domain. This goal is both general, to develop arguments applicable to arbitrary domains, and specific, to study these relations in particular domains. A particular domain may be of interest because of its illustrative significance, or because there are philosophical problems in that domain that can be fruitfully approached by considering the relations between metaphysics and the nature of concepts.

The book formulates and argues for a Primary Thesis:

The metaphysics of a domain is involved in the philosophical explanation of the nature of the meanings of sentences about that domain; and the metaphysics of a domain is involved in the philosophical explanation of the nature of intentional contents (ways of representing) concerning that domain.

Such involvement of the metaphysics can take one of two forms, depending on the domain in question. In metaphysics-first cases, the metaphysics of the domain is drawn upon or presupposed in the account of the nature of concepts of entities in that domain, but not conversely. In no-priority cases, each of the metaphysics and the nature of the concepts involves the other. Each kind of case has instances.

The general, domain-independent argument for the Primary Thesis rests on a certain conception of concepts. A concept, of whatever category, is individuated by the relation that has to hold between a thinker and an entity for the thinker to be thinking of an entity under that concept. Now the relations in which a thinker can stand to an entity are constrained by the metaphysics of that entity. So the metaphysics of an entity constrains ways of thinking about it. The conclusion of this

Christopher Peacocke

cp2161@columbia.edu

1 New York, USA 
argument stands in opposition to meaning-first or concept-first views, plausibly attributable to Dummett and Brandom.

Chapter 1 of $P M$ illustrates the Primary Thesis for perceptual concepts; it draws a contrast with McDowell's thesis of 'The Unboundedness of the Conceptual'; and it emphasizes that the Primary Thesis concerns the order of explanation, not the order of discovery.

The next four chapters of the book illustrate the operation of the Primary Thesis in various particular domains of interest. Doing so involves the consideration, and in some cases the new development, of a substantive metaphysics, the consideration of a substantive theory of concepts of the domain, and consideration of the relations between the two.

Chapter 2 develops a metaphysics of magnitudes, more specifically the extensive magnitudes for which an operation of addition is applicable. It formulates seven principles of a metaphysics of extensive magnitudes. They involve a realism about magnitudes, motivated by arguments that propositions about an extensive magnitudes cannot be reduced to propositions about relations to some standard object or event. Nor can they be captured by propositions about the extensions of various predicates. Extensive magnitudes support an algebra of ratios of them, and this fact can be used to explain how they can feature in causal-explanatory laws. For a perceptual state to be as of a given extensive magnitude, it has to stand in a certain network of relations to extensive magnitudes of a given type. This treatment of magnitudes allows an explanation of analogue computation and analogue representation, and provides materials to answer Kuhn's doubts about the theoryindependence of perceptual content.

The following Chapter considers the Primary Thesis for the special case of temporal magnitudes, and temporal properties and relations. This too is a metaphysics-first case, a long tradition to the contrary having overlooked both the resources available to a metaphysics-first account, and various difficulties inherent in subjectivist accounts of temporal content. The chapter also defends a version of phenomenal externalism about temporal and spatial magnitudes, in contrast to the views of Chalmers and Thompson. The discussion also offers an account of what it is for a mental state to have temporal content, as opposed to being merely sensitive to temporal states of affairs, an account that contrasts with the position of Burge on the issue.

Chapter 4 discusses the ever-problematic topic of the first person concept and its relation to the self. It opens by considering an answer, improved over some of the author's previous attempts, to the question of why mental events have to have a subject. One answer is that perceptual states have the representational content they do in virtue of their significance for future possible actions of the subject. This ties the very nature of perceptual states to the identity of subjects. It also applies to unconscious as well as conscious perceptions, unlike some other accounts. The identity of a subject over time depends on the subject having the same material integration apparatus, that integrates information from perceptual systems into a unified representation of the world, a representation that is drawn upon when the subject engages in intentional action. If this account is correct, it has implications for the 'personites' discussed by Johnston, which are proper temporal segment of 
subjects. The identity over time of a personite depends on the continuing existence of a material integration apparatus. But this apparatus continues after a personite ceases to exist, or exists before the personite does. A personite is not intelligible except as a segment of a genuine subject, and so is ontologically derivative. The first person way of thinking is integrated with an explanatory prior account of subjects by way of a distinctive account of the first person. First person content is to be distinguished from thought merely about the body or about a location. An agencyinvolving account is offered, according to which the capacity for first person content requires ascription of action-properties, which are themselves intrinsic in various ways to being a subject.

Chapter 5 discusses number, a topic not only of interest in itself, but as one which exemplifies the possibility of developing a metaphysics-first treatment without any commitment to causal interaction with the reference of the concepts. The crucial move that opens up this possibility is the stance expressed in the slogan 'Individuation Precedes Representation'. The slogan applies in those cases in which we first have an account of what principles individuate entities of a given kind, and then hold that to grasp certain concepts of those entities is to have tacit knowledge of those individuating principles. In the case of natural numbers, such an account starts from the idea that each natural number $n$ is individuated by the condition for there to be $\mathrm{n}$ things that are $\mathrm{F}$, for arbitrary property $\mathrm{F}$. Then to think of a number as 0 , for instance, is to have tacit knowledge that it is the unique thing $\mathrm{x}$ such that for there to be $\mathrm{x}$ Fs is for it to be the case that nothing is F. This tacit knowledge explains the thinker's assessment of the truth value of contents concerning 0 . This account makes natural numbers objective, and truths about them mind-independent (when applied to mind-independent topics), but nevertheless epistemically accessible. An extension to the case of real numbers draws upon the treatment of magnitudes in Chapter 2. That treatment of magnitudes provides the starting point for a development of the conception of real numbers as ratios of extensive magnitudes. This conception respects the role of real numbers in geometry, in measurement, and in scientific laws.

The final chapter applies the conception of the relation between metaphysics and concepts to provide a new account of the limits of intelligibility. Such alleged entities as absolute space and time, Cartesian egos, quiddities, and certain kinds of possibilia, have been widely recognized as spurious. There has however been very little agreement on the principles to which we should appeal in classifying these entities as spurious. The approach of $P M$ suggests initially a new criterion. For each of these alleged entities, there is, for reasons of the nature of the case, no possible account of what relation would have to hold between a concept as employed by a thinker and one of these entities for the concept to have that entity as its reference. But such a relation is required if there is to be such a genuine concept, for concepts are individuated by such relations. There is an excess dimension in the alleged metaphysics of each of the problematic entities that makes an account of such a relation impossible. This impossibility is a special case of the intrinsic explanatory vacuity of these problematic entities. They lack any explanatory powers that go beyond their less problematic counterparts, such as forms of non-absolute space and time, subjects with identities not tied to Cartesian egos, and the rest. The limits of 
intelligibility are ultimately set not by the bounds of possible experience, but by the bounds of legitimate metaphysics.

Publisher's Note Springer Nature remains neutral with regard to jurisdictional claims in published maps and institutional affiliations. 